\title{
Aggressive Anticoagulation May Decrease Mortality in Obese Critically III COVID-19 Patients
}

\author{
Panagiotis Drakos ${ }^{1}$. Panagiotis Volteas ${ }^{1} \cdot$ Zaina Naeem $^{1}$ (1) $\cdot$ Anthony A. Asencio ${ }^{2} \cdot$ Nathaniel A. Cleri $^{2}$. \\ Leor N. Alkadaa ${ }^{2}$. Anthony Oganov² $\cdot$ Theresa Gammel $^{2}$. Jordan R. Saadon ${ }^{2}$ Mohsen Bannazadeh ${ }^{1,3}$. \\ Apostolos K. Tassiopoulos ${ }^{1,3}$. Charles B. Mikell ${ }^{2}$ - Jerry Rubano ${ }^{1}$ - Aurora Pryor ${ }^{1,4}$ • Konstantinos Spaniolas ${ }^{1,4,5}$. \\ Sima Mofakham ${ }^{2,5}$
}

Received: 17 August 2021 / Revised: 8 November 2021 / Accepted: 10 November 2021 / Published online: 24 November 2021

(c) The Author(s), under exclusive licence to Springer Science+Business Media, LLC, part of Springer Nature 2021

\begin{abstract}
Background Obesity is a widely accepted risk factor for the development of severe COVID-19. We sought to determine the survival benefit of early initiation of aggressive anticoagulation in obese critically ill COVID-19 patients.

Methods We retrospectively reviewed 237 intubated patients at a single academic accredited bariatric center and stratified them based on their BMI into 2 groups, obese $(\mathrm{BMI}>30)$ and non-obese $(\mathrm{BMI} \leq 30)$. We used chi-square tests to compare categorical variables such as age and sex, and two-sample $t$-tests or Mann Whitney $U$-tests for continuous variables, including important laboratory values. Cox proportional-hazards regression models were utilized to determine whether obesity was an independent predictor of survival and multivariable analysis was performed to compare risk factors that were deemed significant in the univariable analysis. Survival with respect to BMI and its association with level of anticoagulation in the obese cohort was evaluated using Kaplan-Meier models.

Results The overall mortality in the obese and non-obese groups was similar at $47 \%$ and $44 \%$, respectively $(p=0.65)$. Further analysis based on the level of AC showed that obese patients placed on early aggressive AC protocol had improved survival compared to obese patients who did not receive protocol based aggressive AC (ON-aggressive AC protocol $26 \%$ versus OFF-aggressive AC protocol $61 \%, p=0.0004$ ).

Conclusions The implementation of early aggressive anticoagulation may balance the negative effects of obesity on the overall mortality in critically ill COVID-19 patients.
\end{abstract}

Keywords COVID-19 Coronavirus · Critically ill $\cdot$ Mechanical ventilation $\cdot$ Body mass index (BMI) - Obesity · Anticoagulation $\cdot$ Heparin $\cdot$ Enoxaparin

Key Points

- The COVID-19 pandemic has led to a global health crisis with $>4$ million deaths.

- Obesity is a risk factor for severe COVID-19 infection and its sequelae.

- In COVID + patients with obesity, early anticoagulation conferred a survival benefit.

Drs. Panagiotis Drakos M.D. and Panagiotis Volteas M.D. contributed equally to this work as first authors. Sima Mofakham Ph.D. and Konstantinos Spaniolas M.D. contributed equally to this work as senior investigators.

Konstantinos Spaniolas

konstantinos.spaniolas@stonybrookmedicine.edu

Sima Mofakham

sima.mofakham@stonybrookmedicine.edu

Extended author information available on the last page of the article

\section{Introduction}

The COVID-19 pandemic, caused by the novel SARSCoV2, has led to a global health crisis with 206 million cases and more than 4.3 million deaths globally [1]. It has been shown that SARS-CoV-2 affects multiple systems causing widespread physiologic insult, including acute respiratory distress syndrome in the context of multiorgan failure, necessitating mechanical ventilation and intensive care unit admission in a significant number of patients [2].

Obesity, defined as a body mass index greater than 30 , is a widely accepted risk factor for the development of severe COVID-19 infection [3-5]. Excess adipose tissue alters underlying hormonal, metabolic and inflammatory processes to adversely influence COVID-19 outcomes in obese 
patients, especially in those under 60 years of age [4]. Significantly, chronic overexpression of both proinflammatory and prothrombotic factors in the setting of obesity exacerbates the inflammatory state induced by SARS-CoV2, thereby predisposing those with concomitant obesity and COVID-19 infection to higher morbidity and mortality risk [6].

In the present study, we hypothesized that patients with obesity would exhibit a more exuberant inflammatory response to COVID-19 infection compared to their nonobese counterparts. We thus sought to investigate whether the implementation of an aggressive anticoagulation (AC) protocol would be able to confer a survival benefit in critically ill COVID-19 patients with obesity.

\section{Methods}

\section{Patient Population and Inclusion Criteria}

This was a retrospective cohort study of all intubated COVID-19 patients, confirmed with a positive RT-PCR test for SARS-CoV2, at a large suburban, academic, accredited bariatric center between February 7, 2020 and May 17, 2020. Our inclusion criteria required patients to be 18 years of age or older and intubated for at least $24 \mathrm{~h}$. We stratified patients based on their BMI into two groups, obese $(\mathrm{BMI}>30)$ and non-obese $(\mathrm{BMI} \leq 30)$. Demographic and clinical characteristics were captured, including gender, age, Sequential Organ Failure (SOFA) score at time of intubation, creatinine, maximum D-dimer level, mortality, steroid administration, and AC.

Clinical care across groups was largely similar, with the exception of AC treatment received. In our institution, we implemented an aggressive AC protocol, which included dose escalation based on daily measured D-dimer levels, as D-dimer is widely used as a biomarker for the hypercoagulable state. Patients with D-dimer $<1000 \mathrm{ng} / \mathrm{mL}$ received enoxaparin $40 \mathrm{mg}$ daily, those with D-dimer $\geq 1000 \mathrm{ng} / \mathrm{mL}$ but $<3000 \mathrm{ng} / \mathrm{mL}$ received enoxaparin $40 \mathrm{mg}$ twice a day and those with D-dimer $\geq 3000 \mathrm{ng} / \mathrm{ml}$ received therapeutic AC with enoxaparin $1 \mathrm{mg} / \mathrm{kg}$ twice a day or intravenous heparin drip at a starting rate of 18 units $/ \mathrm{kg} / \mathrm{h}$ to achieve a goal PTT of 60 to 90 [7]. Choice of anticoagulation agent was determined by the attending physician of record with enoxaparin generally avoided in patients with severe renal impairment. Per the above, we performed a subgroup analysis and stratified the patients with $\mathrm{BMI}>30$ into ON-aggressive $\mathrm{AC}$ (escalation of $\mathrm{AC}$ from once daily to twice daily or to therapeutic AC based on the above D dimer cut-off levels) and OFF-aggressive AC (no escalation of AC according to the D-dimer level driven protocol) and compared for the same demographic and clinical characteristics as in the overall cohort.
Sequential Organ Failure Assessment (SOFA) scores were calculated for all patients at time of intubation, which coincided with ICU admission. The SOFA score is often used in the clinical setting to quantify the degree of organ dysfunction or failure present on ICU admission and aids in predicting ICU mortality risk [8].

The local Institutional Research Board approved the study protocol and supervised all study procedures, in accordance with state and federal regulations, with a waiver of informed consent.

\section{Statistical Analyses}

Statistical analyses were performed using MATLAB and SPSS 21.0 software (SPSS Inc, Chicago, IL). Statistical significance was set at alpha $<0.05$. All reported $p$-values were calculated in a two-sided setting. Data were reported as group means, along with the two-tailed Student's $T$-statistic for several variables of interest. We utilized chi-square tests to compare for any significant difference between two groups for categorical variables (e.g., gender), two-sample $t$-tests for parametric (e.g., age, SOFA) and Mann Whitney $U$-tests for non-parametric continuous variables (e.g., D-dimer, creatinine). Cox proportional-hazards regression models were utilized to determine whether obesity was an independent predictor of survival. Based on the univariable analysis, we determined significant factors to be involved in the multivariable Cox regression model. These factors included age, gender, SOFA score and participation in the D-dimer driven escalation of AC protocol. Entry-level for multivariable analysis was $p$-value $<0.1$. This model provided hazard ratios to estimate which parameters are independent predictors of survival. We assessed the survival of the subgroups using Kaplan-Meier models and a log-rank test. Each patient was followed for a minimum of five months with no missing data points in terms to survival.

\section{Results}

A total of 237 critically ill intubated patients with confirmed COVID-19 infection met inclusion criteria. Five patients were excluded as BMI data were missing. Figure 1 shows the distribution of BMI for this cohort with mean of $30.4 \mathrm{~kg} /$ $\mathrm{m}^{2}$ and SD of 6.33 .

The patient cohort was stratified into obese (BMI $>30$, $n=106)$ and non-obese (BMI $\leq 30, n=131)$ groups. The two groups were similar in age (mean: nonobese $=61.6$, obese $=57.9, p=0.087)$, gender $(70.9 \%$ male in non-obese group versus $68.8 \%$ male in obese group, $p=0.72$ ), SOFA score (mean: non-obese $=6.28$, obese $=6.52, p=0.13$ ), steroid use during hospital course (mean: non-obese $=81.8 \%$ obese $=83.4 \%, p=0.74$ ), and 


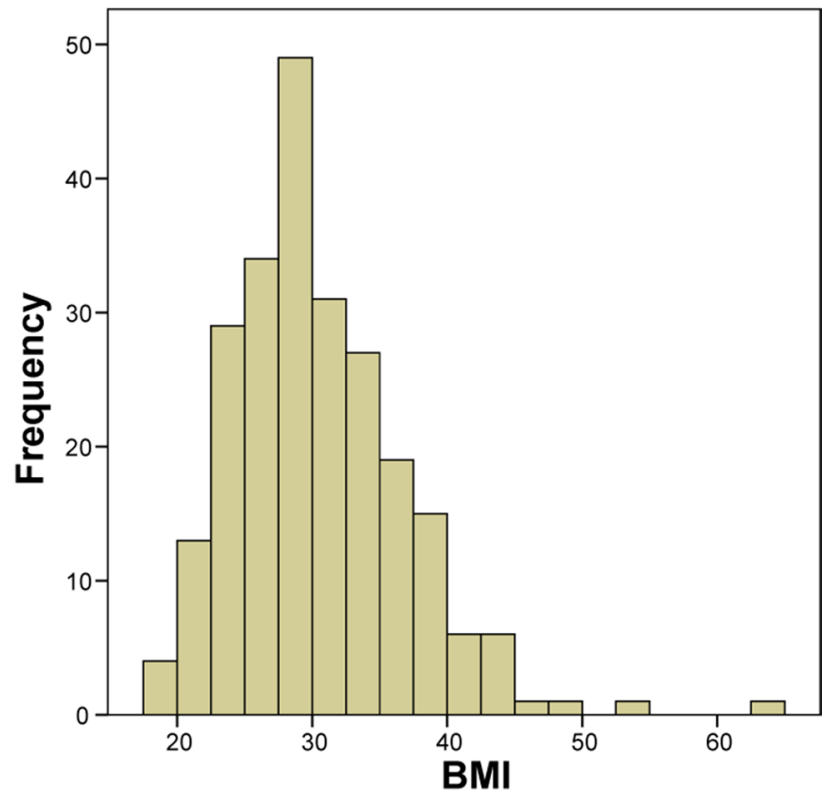

Fig. 1 Distribution of BMI for 237 critically ill, intubated COVID-19 patients

implementation of early aggressive AC protocol (mean: non-obese $=44.8 \%$, obese $=46 \%, p=0.86$ ). Admission creatinine level for patients with obesity was significantly higher compared to their non-obese counterparts (mean: non-obese $=1.28$, obese $=1.38, p=0.027)($ Table 1$)$.
Cox regression for survival multivariate analyses was performed to identify risk factors contributing to mortality in the study population. Factors significantly associated with mortality were age $(\mathrm{HR}=2.482, p<0.0001)$, SOFA score $(\mathrm{HR}=1.856, p=0.005)$, gender $(\mathrm{HR}=1.824, p=0.015)$, and implementation of early aggressive AC (ON-AC protocol) (HR $=0.444, p<0.0001)$ (Table 2). Hypertension, diabetes mellitus, congestive heart failure, chronic kidney disease, chronic obstructive pulmonary disease and BMI were not found to be significant in the univariate analysis and thus were not included in the multivariable survival model.

Kaplan-Meier survival analysis demonstrated no difference in cumulative survival between patients with $\mathrm{BMI} \leq 30$ and those with BMI $>30$ (mortality $44 \%$ versus $47 \%$, $p=0.51$ ) (Fig. 2 left). We further examined the effect of obesity on mortality by stratifying groups at a higher BMI threshold of 35 (severely obese group $n=50$, non-severely obese group $=187$ ), with $50 \%$ mortality in the severely obese group compared to a lower rate of $44.3 \%$ mortality in the non-severely obese group ( $p=0.4$ ) (Fig. 2 center). The cumulative survival was not statistically different using BMI of 35 as a cut-off point. We obtained similar results when using BMI cut-off of 40 (morbidly obese group $n=16$, non-morbidly obese group $n=221$, respectively, $p=0.32$ ) (Fig. 2 right).

As there was no difference in cumulative survival between patients on the basis of BMI, we examined whether the implementation of aggressive AC would offer a beneficial effect in our patient cohort. For patients with

Table 1 Patient demographics

\begin{tabular}{llll}
\hline & BMI $\leq 30(n=131)$ & BMI $>30(n=106)$ & $p$-value \\
\hline Gender (male) & $70.9 \%$ & $68.8 \%$ & 0.72 \\
Age (average, SD) & $61.6+15.31$ & $57.9+13.23$ & 0.087 \\
BMI (average, SD) & $26+2.7$ & $35.8+5.1$ & 0.0001 \\
SOFA score (average) & $6.28+2.25$ & $6.52+2.55$ & 0.13 \\
Creatinine (admission) & $1.28+0.11$ & $1.38+0.14$ & 0.027 \\
Mortality & $44.2 \%$ & $47.1 \%$ & 0.51 \\
Maximum D-dimer & $9504+994$ & $7920+1064$ & 0.12 \\
Implementation of early aggressive anticoagulation based on & $44.8 \%$ & $46 \%$ & 0.86 \\
$\quad$ D-dimer protocol (ON protocol versus OFF protocol) & & & 0.74 \\
Steroids & $81.8 \%$ & $83.4 \%$ \\
\hline
\end{tabular}

Table 2 Multivariable analysis

\begin{tabular}{lllc}
\hline Variable & Comparison level & Hazard ratio & $p$-value \\
\hline Gender & Male vs female & 1.824 & 0.015 \\
Age & More than 70 vs less than 70 years old & 2.482 & $<0.0001$ \\
SOFA & More than 7 vs less than 7 & 1.856 & 0.005 \\
Anticoagulation & Aggressive (ON-AC protocol) vs routine AC & 0.444 & $<0.0001$ \\
& (OFF-AC protocol) & & \\
\hline
\end{tabular}



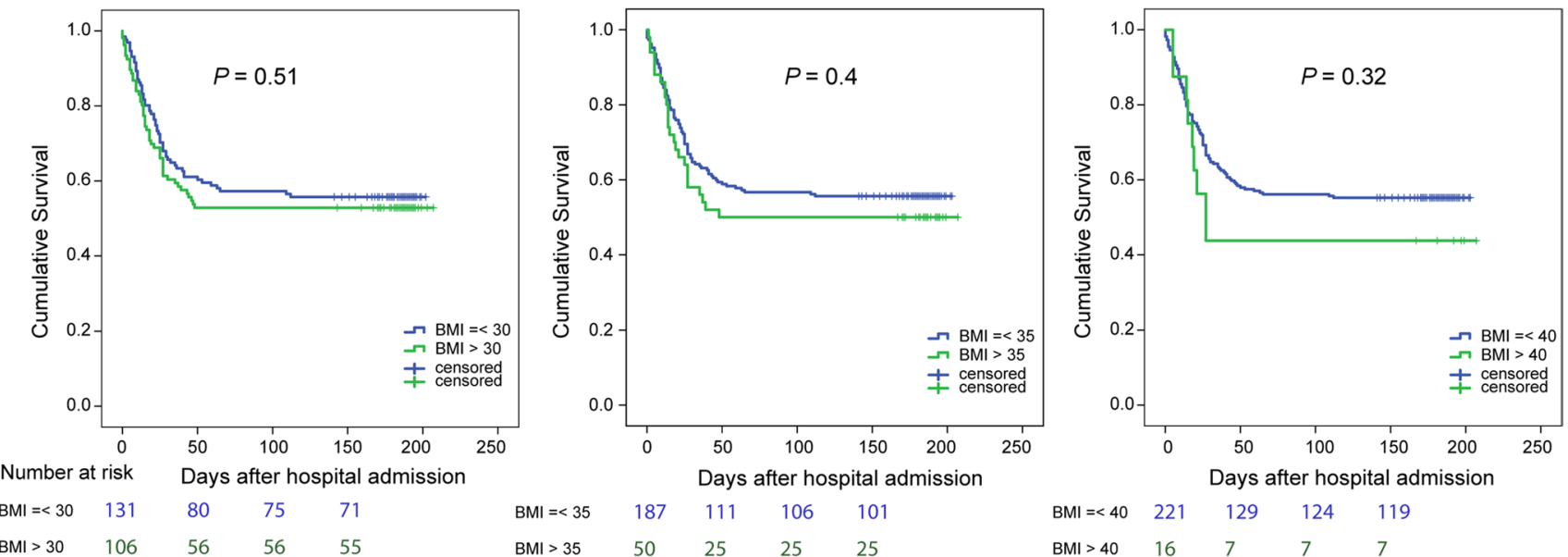

Fig. 2 Comparison of cumulative survival among patients with cut-off BMI 30, 35, and 40

$\mathrm{BMI} \leq 30$, aggressive anticoagulation conferred a survival benefit (OFF-AC mortality $55 \%$ vs. ON-AC mortality $28.5 \%, p=0.002$ ). We then explored whether the same applied to those with obesity, as this cohort analysis has not been previously performed in the literature. After the division of the BMI $>30$ group into $\mathrm{ON}$ - and OFF-aggressive $\mathrm{AC}$ protocol patients, we demonstrated a survival benefit in those who received early aggressive $\mathrm{AC}$ (mortality $\mathrm{ON}$ aggressive $\mathrm{AC}=26 \%$ versus OFF-aggressive $\mathrm{AC}=61 \%$, $p=0.0004$ ) (Table 3 and Fig. 3). Maximum D-dimer level was significantly higher in the OFF-aggressive $\mathrm{AC}$ patients compared to those $\mathrm{ON}$-aggressive $\mathrm{AC}$ patients (6304 versus 9772 respectively, $p=0.024$ ). The small baseline difference in the creatinine that was found in the overall cohort was not appreciated in this subgroup analysis $(p=0.4$, Table 3$)$. No significant difference was noted between ON-AC protocol versus OFF-AC patients with $\mathrm{BMI}>30$ with respect to comorbidities, including CHF ( $p=0.39), \operatorname{CKD}(p=0.46)$, or COPD $(p=0.8)$ (Table 3$)$.

\section{Discussion}

Our study notably found that obesity (defined as a BMI greater than 30) is not associated with higher rates of mortality amongst critically ill intubated patients with COVID19 when compared to those with BMI less than 30. Similar rates of cumulative survival were also observed among study patients when stratified at BMI cutoffs of 35 and 40 . These findings are in contrast with the results of two large meta-analyses which demonstrated significantly higher odds of severe COVID-19 outcomes amongst those with higher BMIs, including hospitalization, invasive mechanical ventilation, and mortality $[9,10]$. Obesity is a well-described risk factor for COVID-19 infection [9-12]. It is widely accepted
Table 3 Characteristics of patients with $\mathrm{BMI}>30$ (ON-AC versus OFF-AC protocol)

\begin{tabular}{llll}
\hline & $\begin{array}{l}\text { ON-AC } \\
\text { protocol } \\
(n=46)\end{array}$ & $\begin{array}{l}\text { OFF-AC pro- } \\
\text { tocol }(n=54)\end{array}$ & $p$-value \\
\hline Gender (male) & $68.5 \%$ & $69.5 \%$ & 0.91 \\
Age (average, SD) & $55.7+11$ & $59.03+15.03$ & 0.12 \\
BMI (average, SD) & $35.4+5.2$ & $36.3+5.3$ & 0.72 \\
SOFA score (average, SD) & $6.08+2.4$ & $6.72+2.5$ & 0.83 \\
Creatinine (admission) & $1.16+0.09$ & $1.43+0.23$ & 0.40 \\
Mortality & $26 \%$ & $61.1 \%$ & 0.0004 \\
Maximum D-dimer (mean, & $6304+1409$ & $9772+1647$ & 0.024 \\
$\quad$ SE) & $84 \%$ & $86 \%$ & 0.76 \\
Steroids & $63 \%$ & $55.5 \%$ & 0.44 \\
$\begin{array}{l}\text { Hypertension } \\
\text { Congestive heart failure }\end{array}$ & $2 \%$ & $5.5 \%$ & 0.39 \\
$\quad$ CHF) & & & \\
$\begin{array}{l}\text { Diabetes, type I or II } \\
\text { Chronic obstructive pulmo- }\end{array}$ & $32.6 \%$ & $20.3 \%$ & 0.16 \\
$\quad$ nary disease (COPD) & $8.6 \%$ & $7.4 \%$ & 0.8 \\
$\quad$ Chronic kidney disease & $4.3 \%$ & $1.8 \%$ & 0.46 \\
$\quad$ (CKD) & & & \\
\hline
\end{tabular}

that obesity increases susceptibility to respiratory infections through various mechanisms, including compromised ventilation in the setting of decreased diaphragmatic excursion, decreased respiratory system compliance, and hyper-circulation of inflammatory cytokines $[11,13]$.

In our subgroup analysis of obese patients with BMI above 30 , we found that those who received early aggressive anticoagulation with therapeutic enoxaparin or heparin drip had approximately half the mortality rate of those receiving the basic thromboprophylaxis regimen (26\% vs $61 \%$ mortality respectively, $p=0.0004$ ), which likely accounted 
Fig. 3 Comparison of cumulative survival among obese patients (BMI > 30) on aggressive anticoagulation $(\mathrm{ON}-\mathrm{AC}$ protocol) versus those receiving routine anticoagulation (OFFAC protocol) $(p<0.001)$

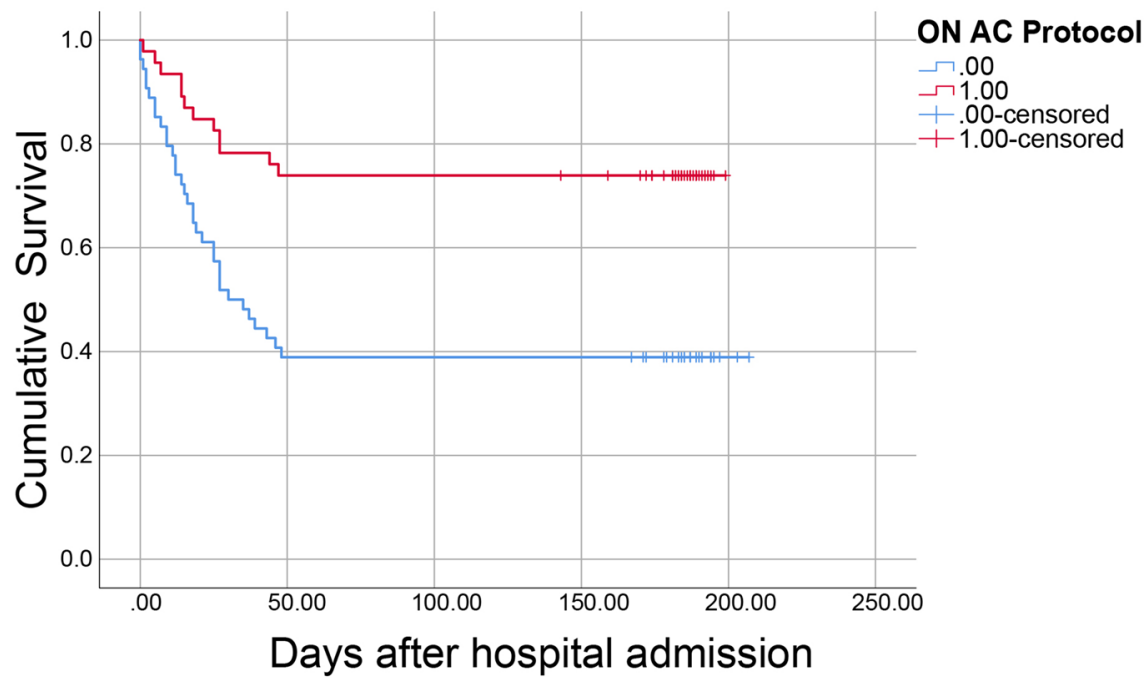

Number at Risk (BMI>30)

\begin{tabular}{|c|c|c|}
\hline ON AC protocol 46 & 34 & 34 \\
\hline OFF AC protocol 54 & 21 & 21 \\
\hline
\end{tabular}

for the discrepancy in mortality benefit observed in this study verses the literature. Anticoagulation benefit has been reported in the general COVID-19 population. Of note, a large retrospective study of 4389 patients in the Journal of the American College of Cardiology describe lower inhospital mortality rates with therapeutic and prophylactic AC (including dual anticoagulant therapy, unfractionated heparin, low molecular weight heparin) compared to no AC (adjusted hazard ratio: 0.53 ; 95\% CI: 0.45 to 0.62 and aHR: 0.50 ; $95 \%$ CI: 0.45 to 0.57 , respectively) [14]. Similarly, in a recent study of 2773 hospitalized patients with COVID-19 in a large New York hospital system, a significantly higher mortality benefit was reported for mechanically ventilated patients who were treated with systemic AC (29.1\% on AC and $62.7 \%$ mortality not on AC) [15].

Surprisingly, there is limited literature examining the risk of thrombosis and the role of AC amongst those with concomitant obesity and COVID-19 infection despite established risk of coagulopathy in each subpopulation alone. Obesity leads itself to a chronic inflammatory state with impaired fibrinolysis, in which hypertrophic adipocytes produce excess proinflammatory cytokines (IL-1 $\beta$, IL-6, IL-8, TNF $\alpha$, and MCP-1) causing upregulation of adhesion molecules and procoagulant factors (notably tissue factor), downregulation of anticoagulant regulatory proteins, increased thrombin generation, and enhanced platelet activation [16-18]. These mechanisms are supplemented by the action of increased circulating levels of free fatty acids through the NF- $\mathrm{kB}$ pathway [18].

It has been proposed that after infecting host cells, SARSCOV-2 also predisposes to a hyperinflammatory reaction through release of a "cytokine storm" amongst other mechanisms, including increased oxidative stress and endothelial dysfunction [18]. COVID-19 coagulopathy is characterized by marked elevations in D-dimer and fibrin fibrin/fibrinogen degradation product levels, placing infected patients at increased risk of arterial and venous thrombosis [19, 20]. Indeed, we found that the maximum D-dimer level was elevated above baseline in our patient population, and significantly more so in the subset not treated with aggressive AC (6304 versus 9772, $p=0.024)$. In an intention-to-treat clinical trial of 3625 COVID-19 patients, higher D-dimer levels were significantly associated with increased mortality; those with D-dimer levels $<1 \mu \mathrm{g} / \mathrm{mL}$ did not appear to benefit from AC while patients with D-dimer levels $>10 \mu \mathrm{g} /$ $\mathrm{mL}$ derived the most benefit [20].

Interestingly, we observed no significant difference in macrovascular thrombotic complications, both venous (DVT, PE) and arterial (myocardial infarction, stroke, peripheral thrombosis), or bleeding complications (upper and lower GI bleed, hemothorax, mediastinal and tracheostomy site bleeding) between ON- versus OFF-AC protocol patients, regardless of BMI. We postulate that given the frequency of pulmonary embolism symptoms in patients with severe COVID-19 infection and the underutilization of imaging in the COVID setting to prevent unnecessary staff exposure, DVT and PE rates may have been underreported in this population.

To our knowledge, this study is the first to examine the role of an aggressive AC protocol in critically ill COVID19 patients with BMI-defined obesity. We notably describe a significant survival benefit amongst obese patients 
attributed to the administration of early aggressive AC regimen, which may compensate for the lack of mortality difference between patients with and without obesity in this study.

\section{Limitations}

This study was a single-center retrospective cohort study and carries with it inherent limitations of this study design, including generalizability. The patient cohort was limited to ventilated patients in the ICU setting and results may not be applicable to COVID patients admitted to lower acuity units. This study did not control for those who had previously been on AC therapy for a pre-existing indication and may therefore have been likely to have better outcomes. A control group consisting of non-anticoagulated patients was not utilized, as all patients were anticoagulated, albeit with different degrees of escalation. Finally, the AC protocol utilized either heparin drip or enoxaparin, which achieve therapeutic effects through different mechanisms of actions and may carry different utility in the critically ill COVID- 19 population, which can be explored in future subgroup analysis.

\section{Conclusions}

This study uniquely proposes that an early aggressive anticoagulation protocol may counteract the negative effects of obesity on the overall mortality in critically ill COVID-19 patients.

Anticoagulation is a modifiable risk factor and represents opportunity for physician intervention, as appropriate.

Funding This work was supported by the SUNY Seed Grant 11607381-87777. No industry or other external funding was used for this research.

\section{Declarations}

Ethics Approval For this retrospective study, formal consent was not required.

Informed Consent Does not apply to this retrospective study.

Conflict of Interest Dr. Tassiopoulos reports personal fees from Medtronic and Terumo, outside the submitted work. Dr. Pryor reports personal fees from Ethicon, Medtronic, Stryker, Gore, Merck and grants from Obalon, outside the submitted work. Dr. Spaniolas reports grants from Merck and personal fees from Gore, outside the submitted work. The remaining authors report no conflicts of interest.

\section{References}

1. COVID-19 Pandemic Weekly Trends. Worldometers. https:// www.worldometers.info/coronavirus/. Last accessed 8/12/21.

2. Berlin DA, Gulick RM, Martinez FJ. Severe Covid-19. N Engl J Med. 2020;383(25):2451-60.

3. Goyal P, Choi JJ, Pinheiro LC, Schenck EJ, Chen R, Jabri A, Satlin MJ, Campion TR Jr, Nahid M, Ringel JB, Hoffman KL, Alshak MN, Li HA, Wehmeyer GT, Rajan M, Reshetnyak E, Hupert N, Horn EM, Martinez FJ, Gulick RM, Safford MM. Clinical characteristics of Covid-19 in New York City. N Engl J Med. 2020;382(24):2372-4.

4. Lighter J, Phillips M, Hochman S, Sterling S, Johnson D, Francois F, Stachel A. Obesity in patients younger than 60 years is a risk factor for COVID-19 hospital admission. Clin Infect Dis. 2020;71(15):896-7.

5. Cai Q, Chen F, Wang T, Luo F, Liu X, Wu Q, He Q, Wang Z, Liu Y, Liu L, Chen J, Xu L. Obesity and COVID-19 severity in a designated hospital in Shenzhen. China Diabetes Care. 2020;43(7):1392-8.

6. Pasquarelli-do-Nascimento G, Braz-de-Melo HA, Faria SS, Santos IO, Kobinger GP, Magalhães KG. Hypercoagulopathy and adipose tissue exacerbated inflammation may explain higher mortality in COVID-19 patients with obesity. Front Endocrinol (Lausanne). 2020;11:530.

7. Tassiopoulos AK, Mofakham S, Rubano JA, Labropoulos N, Bannazadeh M, Drakos P, Volteas P, Cleri NA, Alkadaa LN, Asencio AA, Oganov A, Hou W, Rutigliano DN, Singer AJ, Vosswinkel J, Talamini M, Mikell CB, Kaushansky K. D-Dimer-driven anticoagulation reduces mortality in intubated COVID-19 patients: a cohort study with a propensity-matched analysis. Front Med (Lausanne). 2021;8:631335.

8. Moreno R, Vincent JL, Matos R, Mendonça A, Cantraine F, Thijs L, Takala J, Sprung C, Antonelli M, Bruining H, Willatts $\mathrm{S}$. The use of maximum SOFA score to quantify organ dysfunction/failure in intensive care. Results of a prospective, multicentre study. Working Group on Sepsis related Problems of the ESICM. Intensive Care Med. 1999;25(7):686-96.

9. Huang Y, Lu Y, Huang YM, Wang M, Ling W, Sui Y, Zhao HL. Obesity in patients with COVID-19: a systematic review and meta-analysis. Metabolism. 2020;113:154378.

10. Ho JSY, Fernando DI, Chan MY, Sia CH. Obesity in COVID19: a systematic review and meta-analysis. Ann Acad Med Singap. 2020;49(12):996-1008.

11. Földi M, Farkas N, Kiss S, Zádori N, Váncsa S, Szakó L, Dembrovszky F, Solymár M, Bartalis E, Szakács Z, Hartmann P, Pár G, Erôss B, Molnár Z, Hegyi P, Szentesi A. Obesity is a risk factor for developing critical condition in COVID19 patients: a systematic review and meta-analysis. Obes Rev. 2020;21(10):e13095.

12. Yang J, Hu J, Zhu C. Obesity aggravates COVID-19: a systematic review and meta-analysis. J Med Virol. 2021;93(1):257-61.

13. Dietz W, Santos-Burgoa C. Obesity and its implications for COVID-19 mortality. Obesity (Silver Spring). 2020;28(6):1005.

14. Nadkarni GN, Lala A, Bagiella E, Chang HL, Moreno PR, Pujadas E, Arvind V, Bose S, Charney AW, Chen MD, Cordon-Cardo C, Dunn AS, Farkouh ME, Glicksberg BS, Kia A, Kohli-Seth R, Levin MA, Timsina P, Zhao S, Fayad ZA, Fuster V. Anticoagulation, bleeding, mortality, and pathology in hospitalized patients With COVID-19. J Am Coll Cardiol. 2020;76(16):1815-26.

15. Paranjpe I, Fuster V, Lala A, Russak AJ, Glicksberg BS, Levin MA, Charney AW, Narula J, Fayad ZA, Bagiella E, Zhao S, Nadkarni GN. Association of treatment dose anticoagulation with in-hospital survival among hospitalized patients with COVID-19. J Am Coll Cardiol. 2020;76(1):122-4. 
16. Kwok S, Adam S, Ho JH, Iqbal Z, Turkington P, Razvi S, Le Roux CW, Soran H, Syed AA. Obesity: a critical risk factor in the COVID-19 pandemic. Clin Obes. 2020;10(6):e12403.

17. Samad F, Ruf W. Inflammation, obesity, and thrombosis. Blood. 2013;122(20):3415-22.

18. Korakas E, Ikonomidis I, Kousathana F, Balampanis K, Kountouri A, Raptis A, Palaiodimou L, Kokkinos A, Lambadiari V. Obesity and COVID-19: immune and metabolic derangement as a possible link to adverse clinical outcomes. Am J Physiol Endocrinol Metab. 2020;319(1):E105-9.

19. Gąsecka A, Borovac JA, Guerreiro RA, Giustozzi M, Parker W, Caldeira D, Chiva-Blanch G. Thrombotic complications in patients with COVID-19: pathophysiological mechanisms, diagnosis, and treatment. Cardiovasc Drugs Ther. 2021;35(2):215-29.
20. Billett HH, Reyes-Gil M, Szymanski J, Ikemura K, Stahl LR, Lo Y, Rahman S, Gonzalez-Lugo JD, Kushnir M, Barouqa M, Golestaneh L, Bellin E. Anticoagulation in COVID-19: effect of enoxaparin, heparin, and apixaban on mortality. Thromb Haemost. 2020;120(12):1691-9.

Publisher's Note Springer Nature remains neutral with regard to jurisdictional claims in published maps and institutional affiliations.

\section{Authors and Affiliations}

\section{Panagiotis Drakos ${ }^{1} \cdot$ Panagiotis Volteas ${ }^{1} \cdot$ Zaina Naeem $^{1}$ (1) . Anthony A. Asencio ${ }^{2} \cdot$ Nathaniel A. Cleri $^{2}$. Leor N. Alkadaa ${ }^{2} \cdot$ Anthony Oganov $^{2} \cdot$ Theresa Gammel $^{2} \cdot$ Jordan R. Saadon ${ }^{2} \cdot$ Mohsen Bannazadeh $^{1,3}$. Apostolos K. Tassiopoulos ${ }^{1,3}$. Charles B. Mikell ${ }^{2}$. Jerry Rubano ${ }^{1}$. Aurora Pryor ${ }^{1,4}$. Konstantinos Spaniolas ${ }^{1,4,5}$. Sima Mofakham ${ }^{2,5}$}

1 Department of Surgery, Renaissance School of Medicine, Stony Brook, NY, USA

2 Department of Neurosurgery, Renaissance School of Medicine, Stony Brook, NY, USA

3 Division of Vascular Surgery, Department of Surgery, Renaissance School of Medicine, Stony Brook, NY, USA
4 Division of Bariatric, Foregut, and Advanced Gastrointestinal Surgery, Department of Surgery, Renaissance School of Medicine, Stony Brook, NY, USA

5 Department of Neurosurgery and Department of Surgery, Health Sciences Center T12-064, Stony Brook, NY 11794, USA 